\title{
Hematopoiesis/Erythropoiesis in Myocardial Infarcts
}

\author{
Bruce I. Goldman, M.D., John Wurzel, M.D. \\ Department of Pathology and Laboratory Medicine, Temple University Medical School, \\ Philadelphia, Pennsylvania
}

\begin{abstract}
Extramedullary hematopoiesis occurring in the myocardium has previously only been reported in a single case of a neonate with cyanotic congenital heart disease. Herein we report the incidental discovery of extramedullary hematopoiesis or pure erythropoiesis in four failing adult hearts with myocardial infarction. In two cases, extramedullary hematopoiesis or erythropoiesis was identified in cardiectomy specimens removed at orthotopic heart transplantation; in two other cases, erythropoiesis was found in left ventricular tissue removed at the time of implantation of left ventricular assist devices. Myocardial hematopoiesis/ erythropoiesis was identified based on characteristic light-microscopic findings in routinely processed tissue and was confirmed by immunhistochemistry using monoclonal antibodies to the erythroid cell marker glycophorin A (positive in all cases), the megakaryocyte marker CD61, and the granulocyte marker neutrophil elastase (the latter two markers positive in one case only). None of the four patients had a myeloproliferative disorder or evidence of a myelophthisic process. No hematopoietic elements were identified in 109 cardiectomy specimens without acute or recent infarcts. Myocardial hematopoiesis or erythropoiesis could represent heretoforeunrecognized manifestations of altered cytokine expression in patients with heart failure due to myocardial infarction.
\end{abstract}

KEY WORDS: Extramedullary hematopoiesis, Myocardial infarct, Heart transplant, Mechanical ventricular assistance.

Mod Pathol 2001;14(6):589-594

Copyright (C 2001 by The United States and Canadian Academy of Pathology, Inc.

VOL. 14, NO. 6, P. 589, 2001 Printed in the U.S.A.

Date of acceptance: February 14, 2001.

Address reprint requests to: Bruce I. Goldman, M.D., Department of Pathology and Laboratory Medicine, Temple University Medical School, 3400 N. Broad St., Philadelphia, PA 19140; e-mail: goldmanb@astro. ocis.temple.edu; fax: 215-707-2781.
In recent years, particular emphasis has been placed upon the role of cytokines, both circulating and locally produced, as potentially important mediators of structural and/or functional cardiovascular changes associated with myocardial infarction (MI) (1) and congestive heart failure (CHF) (reviewed in 2). Another pathologic entity associated with altered cytokine expression is extramedullary hematopoiesis (EMH), the production of blood cells outside of the bone marrow. A potential relationship between CHF and EMH is suggested by several case reports documenting EMH, either intracardiac (3) or spinal epidural $(4,5)$, in patients with heart failure associated with cyanotic congenital heart disease. However, myocardial EMH has not been described in adults with heart failure secondary to acquired heart disease. Herein, we describe four cases in which EMH or erythropoiesis was identified in myocardial specimens obtained during the course of surgical treatment for end stage heart failure due to MI, including two cases in which orthotopic cardiac transplantation was performed and two cases in which left ventricular assist devices (LVADs) were implanted.

\section{METHODS}

EMH or pure erythropoiesis was identified in tissue samples received for routine processing in the surgical pathology laboratory of Temple University Hospital. Two specimens were cardiectomies removed at orthotopic transplantation, and two were left ventricular cores obtained at placement of ventricular assist devices. Specimens were received immersion fixed in $10 \%$ buffered formalin. Gross examination, tissue sampling, and preparation of hematoxylin and eosin-stained microscopic sections $(5 \mu \mathrm{m})$ was performed according to standard protocols. Immuohistochemical staining was performed on formalin-fixed, paraffin-embedded tissues using monoclonal antibodies to glycophorin A (specific for erythroid cells), CD61 (specific for megakaryocytes), and neutrophil elastase (specific for granulocytes). All antibodies were obtained from DAKO Laboratories (Carpinteria, CA); titers 
were empirically determined using control tissues. Staining used a commercially available avidinbiotin-peroxidase complex method and was performed with an automated stainer (DAKO). Negative controls used irrelevant monoclonal antibodies. Enzyme histochemistry was not performed.

\section{Clinical Histories}

\section{Case 1}

A 28-year-old female with history of restrictive cardiomyopathy for 10 years, hypercoagulable state treated with warfarin and aspirin, and remote pulmonary thromboembolism and stroke without permanent deficit underwent orthotopic heart transplantation for intractable heart failure (ejection fraction, 25-35\%). Examination of the explanted native heart showed biventricular hypertrophy, diffuse myocardial interstitial fibrosis, and coronary atherosclerosis; Congo red and crystal violet stains showed no myocardial amyloid. The postoperative course was characterized by severe graft dysfunction, necessitating placement of a biventricular assist device (Abiomed, Danvers, MA), persistent thrombocytopenia with oozing from multiple sites necessitating repeated blood product transfusions, and intermittent respiratory insufficiency with hypoxia. Retransplantation was performed after 14 days. Hemoglobin, hematocrit, white blood cell count, and platelet count at the time of first transplantation were normal. Between the first and second transplants, hemoglobin levels ranged between $70 \mathrm{~g} / \mathrm{L}$ and $95 \mathrm{~g} / \mathrm{L}$, and white blood cell count rose as high as $40 \times 10^{12}$ per liter, with a left shift.

\section{Case 2}

A 29-year-old male with Type I diabetes mellitus for 20 years, hypercholesterolemia, hypertension, renal insufficiency, coronary artery bypass surgery 2 years before admission, and recent MI underwent orthotopic heart transplantation for intractable severe heart failure (ejection fraction, 15\%). White blood cell count had risen as high as $22 \times 10^{12}$ per liter in the week before transplantation but eventually normalized after surgery. Hemoglobin and hematocrit had been depressed before transplantation (hemoglobin level, approximately 100-110 $\mathrm{g} / \mathrm{L}$ ), with normal red cell parameters. Anemia was believed to represent effect of chronic disease, and no transfusion was given before transplantation.

\section{Case 3}

A 61-year-old male with history of MI, percutaneous transluminal coronary angioplasty, hypertension, hypercholesterolemia, and mild renal insufficiency underwent emergent coronary artery bypass grafting for unstable angina. He was unable to be weaned from bypass, was placed on a biven- tricular assist device (Abiomed), and was transferred to our institution to be evaluated for cardiac transplantation. At admission, the hemoglobin was $92 \mathrm{~g} / \mathrm{L}$, with normal red cell parameters. The hospital course was complicated by bleeding from operative sites, with hemoglobin levels dropping as low as $72 \mathrm{~g} / \mathrm{L}$, thrombocytopenia felt due to biVAD placement, Gram-negative pneumonia treated with Cefipine and vancomycin, renal insufficiency, and hyperbilirubinemia with increased ALT and AST felt to be drug induced hepatitis. Abiomed biVAD was replaced on Hospital Day 13 with an implantable LVAD (ThermoCardiosystems [TCI]), but the patient died shortly after surgery. A thoracoabdominal autopsy was performed.

\section{Case 4}

A 58-year-old male with atherosclerotic coronary artery disease and inferior wall MI 16 years before admission presented to his local hospital with signs and symptoms of acute MI. Thrombolysis resulted in initial resolution of symptoms, but acute lateral wall MI developed 30 minutes later. Emergent coronary artery bypass was performed, but intraoperative cardiogenic shock necessitated placement of an LVAD (Abiomed). The postoperative course was complicated by aspiration pneumonia and mediastinitis, and he was transferred to our institution for chest closure and transplant evaluation. Pneumonia and mediastinitis resolved with treatment, and a fully implantable LVAD (TCI) was placed because of the need for prolonged ventricular support. The post-LVAD course was complicated by recurrent bleeding from implantation sites and renal failure, and the patient died 1 day after LVAD placement. No autopsy was performed.

\section{RESULTS}

\section{Case 1}

The explanted cardiac allograft weighed $179 \mathrm{~g}$. The epicardium showed diffuse fibrinohemorrhagic pericarditis. The myocardium showed a zone of grossly evident recent hemorrhagic infarction involving postero-inferior left ventricle, posterior right ventricle, and adjacent interventricular septum (Fig 1A). Microscopy confirmed recent biventricular infarction with granulation tissue and early fibrosis, consistent with infarct age of 2 weeks. The coronary arteries showed no significant stenoses or thrombi. No bone marrow emboli were seen.

Within the peri-infarct granulation tissue were islands of hematopoietic cells, including clusters of erythroblasts, granulocytic precursors, and megakaryocytes (Fig. 1, B-C). The presence of hematopoietic precursors of all three cell types was confirmed by immunohistochemical staining of 
characteristic cell types (Fig. 1, D-F.) Mild acute cellular and humoral rejection were also present (not shown).

\section{Case 2}

The explanted heart weighed $377 \mathrm{~g}$. There was significant multivessel calcific coronary atherosclerosis as well as four separate distal segments of coronary artery bypass grafts showing stenosis by old mural thrombosis and fibrointimal hyperplasia. No bone marrow emboli were seen. The myocardium exhibited healing transmural infarction involving anterior walls of both ventricles and contiguous interventricular septum, as well as extensive transmural scarring of the left ventricular apex and anterior wall. Granulation tissue adjacent to the healing infarct contained islands of erythropoiesis (Fig. 1, G), confirmed by immunostaining with antibody to glycophorin A (Fig. 1, H). No granulocytopoiesis or megakaryopoiesis was identified.

\section{Cases 3 and 4}

Specimens were LVAD cores measuring approximately $1.5 \mathrm{~cm}$ in greatest dimension. Microscopic examination of both samples showed recent MI, with zones of necrosis bordered by granulation tissue. Islands of erythroblasts were seen in the epicardial fat of Case 3 and in the peri-infarct granulation tissue of Case 4. No bone fragments nor marrow emboli were identified. Immunohistochemical staining confirmed extramedullary erythropoiesis (in other words, nucleated cells with glycophorin A positivity) in both cases (not shown). No megakarypoiesis nor granulopoiesis was found in either case.

\section{DISCUSSION}

This report is the first to document myocardial EMH or erythropoiesis in adult hearts with acquired disease. EMH/erythropoiesis was identified by the characteristic light-microscopic appearance of islands of hematopoietic cells in hematoxylin and eosin-stained sections and was confirmed by immunostaining for hematopoietic cell markers. Erythropoiesis was identified in all four cases; concomitant granulopoiesis and megakaryopoiesis were found in one case only. No patient had clinical evidence of primary hematologic disease or myelophthisic process. In all instances, EMH or erythropoiesis was found adjacent to zones of MI, a feature that may have relevance to the pathogenesis of EMH in these hearts.

Myocardial EMH has only rarely been described. A search of the literature from the last 30 years yielded only one reported case in which EMH was found in the heart of a neonate with cyanotic congenital heart disease due to tricuspid atresia (3). That patient had undergone placement of an aortopulmonary fistula 1 day after birth and developed persistent postoperative pericardial effusion. Pericardial EMH was diagnosed by cytologic examination of pericardial fluid, and EMH involving multiple sites including the heart was confirmed at autopsy $<2$ weeks after pericardiocentesis. Two other cases of EMH associated with heart failure but not involving the heart also have been reported. In both of these cases, patients had cyanotic congenital heart disease and presented with paraparesis due to epidural involvement by $\operatorname{EMH}(4,5)$.

Although myocardial EMH has only rarely been reported, our experience suggests it may be relatively common, at least in hearts with MI severe enough to prompt transplant or LVAD use. The four cases reported here were found among 11 total surgical pathology accessions received since mid1998 showing myocardium with acute or recent MI. The frequency of myocardial EMH observed in infarcted myocardium in our experience is thus $37 \%$ $(4 / 11)$. Our experience further suggests that myocardial EMH/erythropoiesis is specifically confined to hearts with acute or recent MI. Since the identification of the index case, one of us (BG) has reviewed 117 surgical cardiectomy specimens, most with diagnostic features of dilated cardiomyopathy or chronic atherosclerotic heart disease. Of these, 109 showed no acute or recent MI and no lightmicroscopic evidence of EMH or erythropoiesis.

The reasons that EMH/erythropoiesis in infarcted myocardium has not been previously reported are not clear. The absence of previous description may simply represent a tendency for pathologists to misidentify foci of EMH as chronic inflammation. Alternatively, the presence of $\mathrm{EMH}$ or erythropoiesis in infarcted myocardium may reflect effects of recent changes in treatment and/or in the natural history of treated MI.

The pathogenesis of EMH or erythropoiesis in infarcted myocardium is also not known, but there are several potential explanations for its development. In general, EMH is seen in myelophthisic diseases or as a response to tissue hypoxia, for example, such as in chronic anemia or hypoxemia. None of our patients had a primary hematologic or marrow-infiltrating disease. However, all of our patients were anemic before cardiac transplantation or assist device placement, although anemia in $\mathrm{Pa}-$ tient 1 was only present for 14 days. Intermittent hypoxemia, another potential stimulus for EMH, was also documented in three of our four cases.

Surgery-related marrow embolization and/or implantation has also been proposed as a cause of EMH. Such a mechanism could explain the development of EMH in all of our cases, because median 

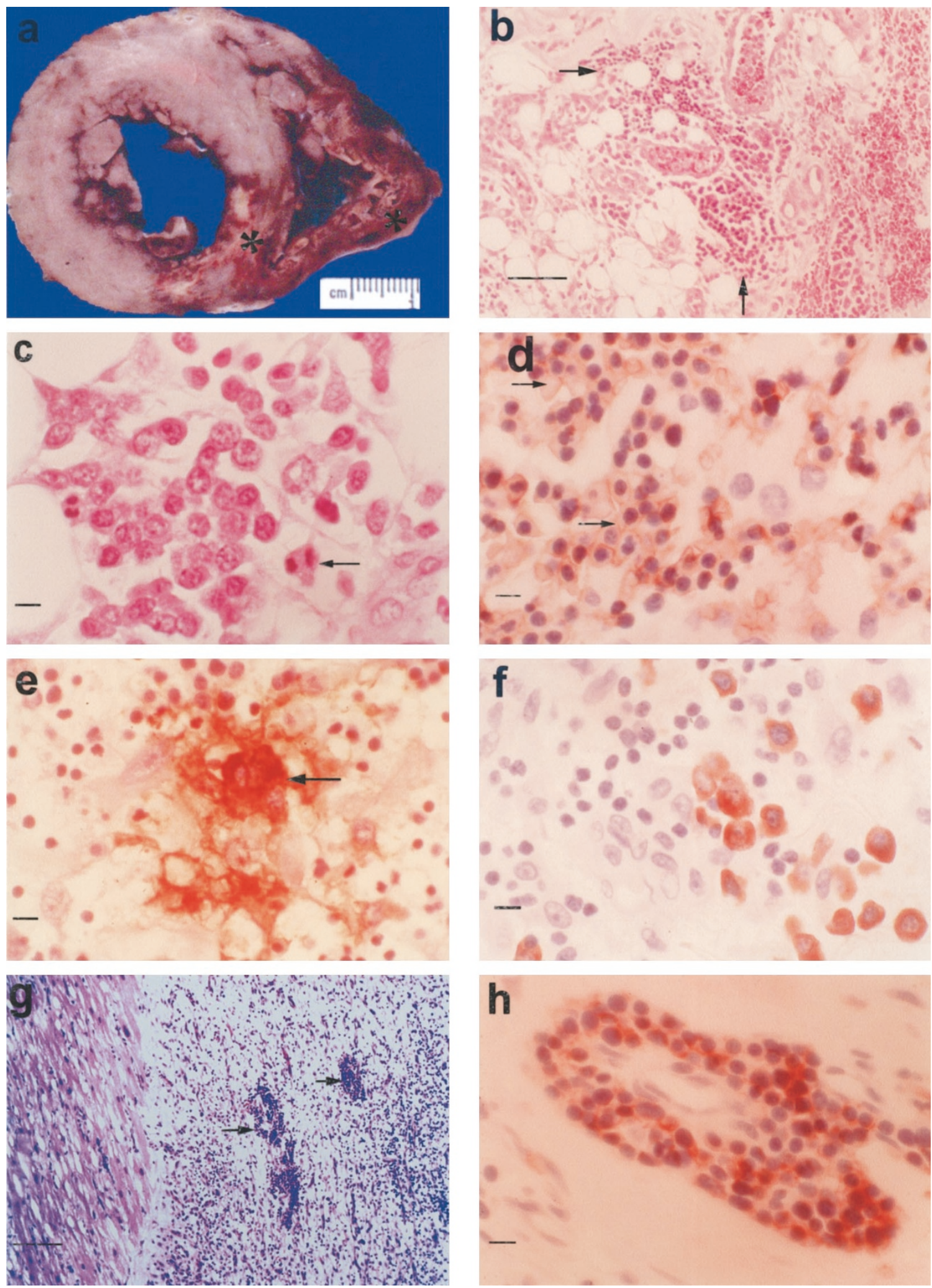

FIGURE 1. Gross and microscopic findings, Cases 1 and 2. A, gross photograph of horizonal section of heart, Case 1. There is extensive hemorrhagic infarction involving posterior walls of both ventricles and adjacent interventricular septum. B, photomicrograph showing focus of extramedullary hematopoiesis, Case 1. Erythroblasts (horizontal arrow) show characteristic small opaque nuclei and scant eosinophilic cytoplasm; myeloblasts (vertical arrow) appear larger with more abundant cytoplasm (hematoxylin and eosin, original magnification, $50 \times$; bar $=100 \mu \mathrm{m}$ ). C, high-power view of myocardial hematopoiesis, Case 1. A cluster of blasts includes some with mitotic figures (arrow; hematoxylin and eosin, original magnification, 200×; bar $=10 \mu \mathrm{m}$ ). D, Same field as B, Case 1, showing erythroblasts with cytoplasm positive for glycophorin A (arrows; all immunostains avidin/biotin/peroxidase/hematoxylin; original magnification 200×; bar $=10 \mu \mathrm{m}$ ). E, anti-CD61 immunostain, Case 1, showing positive megakaryocytes (arrow). F, anti-neutrophil elastase immunostain, Case 1, showing granulocytic precursors with cytoplasmic positivity (right side of photograph). G, photomicrograph of healing infarct, Case 2, showing foci of erythropoiesis (arrows; hematoxylin and eosin, original magnification, $50 \times$; bar $=100 \mu \mathrm{m})$. $\mathbf{H}$, same focus as $\mathbf{G}$, Case 2, showing erythroblasts stained with anti-glycophorin A. 
sternotomy had been performed in Patient 1 for heart transplantation and in Patients 2-4 for coronary artery bypass procedures. Of interest in this regard is that in the only other reported case of cardiac EMH, cardiac surgery had also been performed before the demonstration of EMH (3). However, in none of our cases did we identify bone or marrow fragments within myo- or epicardium or marrow emboli within the microvasculature. Marrow embolization occurring in the remote past might be evident as intravascular fat necrosis, which also was not identified. The absence of fat necrosis, bone fragments, or marrow emboli, even when the interval between previous cardiac surgery and the tissue identification of EMH was as short as 2 weeks (Cases 1, 3, and 4) suggests that marrow emboli or implants acquired in previous surgery probably do not explain the pathogenesis of the myocardial EMH we observed.

Although marrow embolization/implantation was not demonstrated in our cases, surgical intervention may have been otherwise related to the development of EMH or erythropoiesis in three of our four patients. In Patient 1, heart transplantation with institution of immunosuppression had occurred 2 weeks before the identification of EMH; in Patients 1, 3, and 4, mechanical ventricular assistance had been in place for nearly 2 weeks before the diagnosis of EMH. The specific mechanisms whereby these interventions may have promoted EMH are not clear, but systemic immunosuppression in Case 1 and systemic effects of mechanical ventricular assistance in all three cases could have been involved. Of potential significance in this regard is that Patient 1 was the only one in which all three hematopoietic cell lines were observed and also the only patient to whom systemic immunosuppression was given. Although immunosuppressants are generally held to be antiproliferative, the findings in Patient 1 suggest that there may be a relationship between the effects of pharmacologic immunosuppression and the development of myocardial EMH.

Another potential mechanism for EMH/erythropoiesis in our cases relates to the presence of altered cytokine profiles in patients with heart failure. Interleukin-6 (IL-6) is a pleiotropic cytokine involved in inflammation, the immune response, hematopoiesis, and neoplasia (reviewed in 6, 7). It is a member of a family of cytokines that act through the gp130 signal transucer glycoprotein $(8,9)$. Elevated plasma levels of IL- 6 have been described in a variety of diseases, including CHF. Plasma IL-6 is elevated in $\mathrm{CHF}$, as are concentrations of the soluble IL-6 receptor (sIL-6-R) and receptor/ligand complexes (10) Schirmacher et al. have demonstrated augmented EMH in transgenic mice overexpressing both IL-6 and sIL-6R, but not in those overexpressing IL-6 alone (11). This observation likely reflects the induction of IL- 6 activity via activation of gp130 by IL-6/sIL-6R complexes (12). Thus, EMH in CHF may represent a heretoforeunrecognized manifestation of concomitant elevations in circulating IL-6 and its receptor.

That all of our cases of cardiac EMH were found in association with recent MI also may be relevant to pathogenesis. Changes in circulating levels of IL-6 and sIL-6R have been shown in patients with acute MI (13). In a rat model of MI, Gwechenberger et al. (14) recently showed elevated expression of IL-6 in the border zone of reperfused infarcts. Taken together, these reports suggest that the microenvironment of healing infarcts may be especially suited to the support of EMH, particularly as regards availability and activity of IL-6.

EMH in three of four cases was manifest as erythropoiesis only, whereas in one case, all hematopoietic cell lines were evident. This difference among case phenotypes has several potential explanations, all of which pertain to the microenvironment of the peri-infarct zone. The preponderance of erythropoiesis in our cases could reflect the presence of numerous macrophages in the infarct, since macrophages have been shown to promote erythropoiesis (15). The peri-infarct zone also may vary in its content of specific extracellular regulators of hemodifferentiation. Granulopoiesis, for example, is regulated by multiple cytokines, matrix components, and enzymes produced by multiple cell types (reviewed in 16). Analogously, erythropoiesis has been shown experimentally to be potentiated by androgens (17) and cytokines, including stem cell factor and erythropoietin (18). Variations in local concentration of cytokines or other growth factors thus also could account for the variable EMH phenotypes we observed.

Although myocardial EMH in MI appears to be a novel finding, it should be noted that EMH has been described in some cases of cardiac myxoma (19). Of note, expression of IL-6 also has been observed in some cases of this neoplasm $(20,21)$. EMH in cardiac myxomas thus also could be related to local production of IL-6.

The significance of EMH in native hearts of patients who are candidates for cardiac transplantation is unclear; however, two aspects deserve mention. First, the report of Luban et al. (3) suggests that myocardial EMH may be a cause of significant pericardial effusion. Second, identification of myocardial EMH or erythropoiesis in myocardial biopsy or cardiectomy specimens should not be taken as evidence of myelophthisic or myeloproliferative disease, because none of our patients had such a condition. In the absence of pericardial effusion or hematologic disorder, EMH in hearts failing with MI may represent an epiphenomenon of little clin- 
ical significance or, alternatively, a manifestation of a previously unrecognized but important aspect of heart failure.

\section{NOTE ADDED IN PROOF}

Subsequent to the preparation of this manuscript, similar findings have been reported by Hill and Swanson in a retrospective survey of 805 myocardial samples submitted for pathologic examination (Hill DA, Swanson PE. Myocardial extramedullary hematopoiesis: a clinicopathologic study. Mod Pathol 2000:13:779-87.)

Acknowledgments: The authors thank Ms. Donna Johns and Mr. Frank Lazzarro for expert assistance with immunohistochemical staining.

\section{REFERENCES}

1. Pudil R, Pidrman V, Krejsek J, Gregor J, Tichy M, Andrys C, et al. Cytokines and adhesion molecules in the course of acute myocardial infarction. Clin Chim Acta 1999;280:127-34.

2. Blum A, Miller H. Role of cytokines in heart failure. Am Heart J 1998;135:181-6.

3. Luban NLC, Kapur S, De Palma L. Pericardial extramedullary hematopoiesis in a neonate with congenital heart disease: a case report. Acta Cytol 1993;37:729-31.

4. Bree RL, Neiman HL, Hodak JA, Flynn RE. Extramedullary hematopoiesis in the spinal epidural space. J Can Assoc Radiol 1974;25:297-9.

5. Taylor CL, Maynard F, Liebman J, Hlavin ML. Extramedullary hematopoiesis causing paraparesis in congenital cyanotic heart disease. Neurology 1998;51:636-7.

6. Hirano T. Interleukin 6 and its receptor: ten years later. Int Rev Immunol 1998;16:249-84.

7. Hirano T, Kishimoto T. Interleukin-6. In: Sporn MB, Roberts $\mathrm{AB}$, editors. Peptide growth factors and their receptors I. New York: Springer-Verlag; 1990. p. 633-65.

8. Fukada T, Yoshida Y, Nishida K, Ohtani T, Shirogane T, Hibi M, et al. Signaling through Gp130: toward a general scenario of cytokine action. Growth Factors 1999;17:81-91.
9. Hirano T, Ishihara K, Hibi M. Roles of STAT3 in mediating the cell growth, differentiation and survival signals relayed through the IL-6 family of cytokine receptors. Oncogene 2000;19:2548-56.

10. Aukrust P, Ueland T, Lien E, Bendtzen K, Muller F, Andreassen AK, et al. Cytokine network in congestive heart failure secondary to ischemic or dilated cardiomyopathy. Am J Cardiol 1999;83:376-82.

11. Schirmacher P, Peters M, Ciliberto G, Blessing M, Lotz J, Meyer zum Buschenfelde KH, et al. Hepatocellular hyperplasia, plasmacytoma formation, and extramedullary hematopoiesis in interleukin (IL)-6/soluble IL-6 receptor doubletransgenic mice. Am J Pathol 1998;153:639-48.

12. Mackiewicz SH, Heinrich PC, Rose-John S. Complex of soluble human IL-6 receptor/IL-6 up-regulates expression of acute phase proteins. J Immunol 1992;149:2021-7.

13. Ueda K, Takahashi M, Ozawa K, Kinoshita M. Decreased soluble interleukin-6 receptor with acute myocardial infarction. Am Heart J 1999;138:908-15.

14. Gwechenberger M, Mendoza LH, Youker KA, Frangiogiannis NG, Smith CW, Michael LH, et al. Cardiac myocytes produce interleukin-6 in culture and in viable border zone of reperfused infarctions. Circulation 1999;99:546-51.

15. Sadahira Y, Mori M. Role of the macrophage in erythropoiesis [review]. Pathol Int 1999;49:841-8.

16. Ward AC, Loeb DM, Soede-Bobok AA, Touw IP, Friedman $\mathrm{AD}$. Regulation of granulopoiesis by transcription factors and cytokine signals [review]. Leukemia 2000;14:973-90.

17. Saitoh T, Morimoto K, Kumagai T, Tsuboi I, Aikawa S, Horie T. Comparison of erythropoietic response to androgen in young and old senescence accelerated mice. Mech Ageing Dev 1999;109:125-39.

18. Morgan DA, Class R, Soslau G, Brodsky I. Cytokine-mediated erythroid maturation in megakaryoblastic human cell line HU-3. Exp Hematol 1997;25:1378-85.

19. Burke AP, Virmani R. Cardiac myxoma. A clinicopathologic study. Am J Clin Pathol 1993;100:671-80.

20. Takizawa T, Sumino H, Kanda T, Kobayashi I, Nagai R, Ichikawa S. An interleukin-6-producing cardiac myxoma associated with mediastinal lymphadenopathy. Cardiology 1999;92:275-7.

21. Hovels-Gurich HH, Seghaye MC, Amo-Takyi BK, Hugel W, Duchateau J, von Bernuth G. Cardiac myxoma in a 6-yearold child-constitutional symptoms mimicking rheumatic disease and the role of interleukin-6. Acta Paediatr 1999;88: $786-8$. 